\title{
The Role of Adenovirus in Respiratory Tract Infections
}

\author{
Anjali N. Kunz $\cdot$ Martin Ottolini
}

Published online: 24 February 2010

(C) 2010 US Government

\begin{abstract}
Adenovirus plays a significant role in respiratory tract disease in pediatric and adult patients. It has been linked to outbreaks and epidemics in various patient populations, resulting in considerable morbidity and mortality. In this article, we discuss the epidemiology, pathogenesis, respiratory tract illnesses and complications, and roles of potential treatment options. The role of the past oral adenovirus vaccine and the military implications of its withdrawal from routine use in military recruits is discussed as well.
\end{abstract}

Keywords Adenovirus - Respiratory tract infections ·

Acute respiratory disease $\cdot$ Conjunctivitis

\section{Introduction}

Adenovirus is an important, yet somewhat enigmatic, pathogen in respiratory tract infections. For many years prior to its identification, it was known to have the greatest impact on young, healthy men during the initial weeks of their military basic training. It was the primary agent responsible for predictable outbreaks of acute respiratory illness in military recruits and was referred to as the "acute respiratory disease of recruits" (ARD) syndrome [1]. After many years of causing significant morbidity, especially in this population, the causative agent was finally identified in

A. N. Kunz $(\triangle) \cdot$ M. Ottolini

Department of Pediatrics,

Uniformed Services University of the Health Sciences,

Bethesda, MD 20184, USA

e-mail: akunz@usuhs.mil

\section{Ottolini}

Infectious Disease Clinical Research Program,

Uniformed Services University of the Health Sciences,

Bethesda, MD 20184, USA
1953 by investigators at the National Institutes of Health $(\mathrm{NIH})$ and by military infectious disease researchers $[1,2]$.

Adenovirus was first isolated from adenoidal tissue and was shown to have a unique cytopathic effect in epithelial cell culture [2, 3]. Since its initial isolation, further investigations have led to isolation of the virus from a variety of tissue sites and in different clinical disease entities. This work has contributed to our understanding of the unique life-cycle of adenovirus.

Currently, the most common recognizable clinical presentations of adenovirus infection are pneumonia, bronchitis/bronchiolitis, conjunctivitis, pharyngoconjunctival fever, and upper respiratory tract infections or common cold symptoms $[2,3]$. It has also been a causative agent in epidemic outbreaks of conjunctivitis, acute lower respiratory tract infections, and febrile pharyngoconjunctival syndromes [1-3]. Six species of adenoviruses have been identified, which contain 51 serotypes [2, 3]. Certain clinical presentations of adenoviral respiratory tract infections are linked to particular serotypes.

In this review, we focus on the most clinically relevant serotypes that cause respiratory tract infections. We also cover the pathogenesis of the virus, common clinical manifestations in the respiratory tract, complications, and management in pediatric and adult patients.

\section{Virus Structure}

The outer surface structure and genetic makeup of the virus play critical roles in its pathogenicity. The outer structure consists of a protein coat, called a capsid, which consists of hundreds of capsomeres [2]. It is arranged into an icosahedral structure which contains three distinct and antigenic geometric elements, a total of 240 hexons, 12 
pentons at the corners, and 12 fibers that protrude from the pentons [2]. It is a simple yet very elegant structure, much like Fuller's geodesic dome, as illustrated in Fig. 1.

The fibers assist with viral attachment and absorption into the cell. The attachment receptors on the cell surface are called coxsackieadenovirus receptors, and they are used by all subgroups except B [2]. The genetic material of the adenovirus is encased in a protein shell that helps maintain its genetic integrity and assists with enzymatic reactions [2].

During all types of adenovirus infection, the virion produces a specific protein called T-antigen. The T-antigen is a macromolecule or early protein thought to play some functional role in biosynthesis of the virion [1-3]. It is produced prior to viral structural proteins and does not require viral DNA replication; any role it may have in oncogenic transformation is unclear [2-4]. Complement fixation and immunofluorescent assays are used to detect this protein, and hence adenovirus, even in the absence of acute infection [2-4].

\section{Pathogenesis}

Many of the common respiratory viruses that cause upper and lower tract disease (eg, influenza, respiratory syncytial virus, parainfluenza, and corona viruses) are enveloped, single-stranded RNA viruses within the families Orthomyxoviridae, Paramyxoviridae, and Coronaviridae [2]. In contrast, adenoviruses are nonenveloped DNA viruses, which belong to the very different family Adenoviridae [2]. The main structural difference is the lack of a hostderived envelope, which enhances their ability to evade destruction by detergents or alcohol-based cleaning agents,

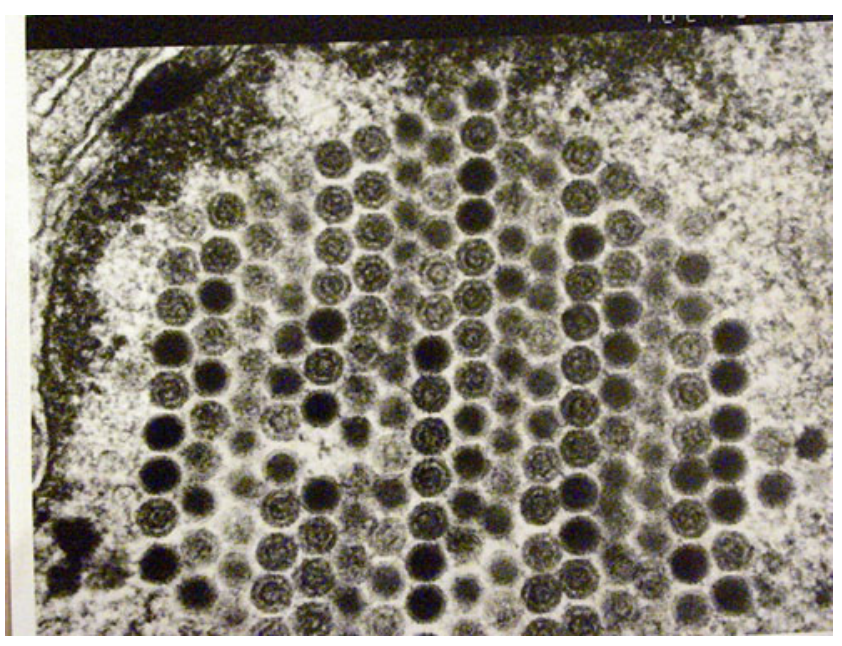

Fig. 1 Electron micrograph picture of Adenovirus serotype 4-infected cell (magnification $\times 108,000$ ). The picture illustrates the multiple adenovirus particles arranged with geometric symmetry within an infected cell. (Courtesy of Ludmila Asher, MD, and Leonard Binn, $\mathrm{PhD}$, at Walter Reed Army Institute of Research.) plays a key role in epidemic outbreaks, and poses a challenge to infection-control measures [2].

The family Adenoviridae contains six subgroups-A, B, $\mathrm{C}, \mathrm{D}, \mathrm{E}$, and $\mathrm{F}$ - which are further subdivided into 51 serotypes [2, 3]. Some of these subgroups have been directly linked to different clinical syndromes, but more than half of the serotypes have not yet been identified with a clinical disease process [2]. This is largely because adenovirus infections are responsible for three distinct types of infection of human cells: lytic infection, latent (chronic) infection, and oncogenic transformation of cells [2, 3]. The lytic process is a fairly rapid sequence of events beginning with active infection of epithelial cells, replication of the virus, and subsequent release of viral progeny with resultant cell death. Only about $1 \%$ to $5 \%$ of the progeny are infectious and therefore able to continue the viral life-cycle. This type of infection is responsible for most of the classic respiratory and enteric clinical illnesses [2, 3]. Latent infection usually occurs in lymphoid tissue and results in a chronic, indolent infection, which is frequently asymptomatic and therefore hard to link to clinical disease. During this process, only a few viral particles are released, and host tissues continue cell replication with minimal to no injury and therefore viral infection is sustained at a very low level in the host $[2,3]$. The last type of infection, oncogenic transformation, has been the main focus of adenoviral research, mostly because of its potential use in future gene therapy and other therapeutics. During this infection, the virus invades human cells and integrates into the cellular genome without production of infectious viral progeny. This may lead to a subsequent oncogenic transformation of the cell, without an acute clinical infection [1-3].

Oncogenic transformation is a unique feature of adenovirus that was initially noted by NIH investigators during early research into the development of an adenovirus vaccine. During these studies, they found that the oncogenic simian virus 40 (SV40) could transform adenovirus 7 into a virus with oncogenic potential $[1,2]$. Furthermore, a variety of studies showed the presence of adenovirus in various human malignancies, although establishing a causal relationship to human cancers has been difficult. Studies by Green, through the NIH, investigated the presence of oncogenic adenovirus in a variety of human tumor cells [1]. These investigations failed to show a link between the virus and development of cancer in humans [1]. However, these studies led to future applications and the development of the live adenovirus vaccine.

Despite being very different processes, all three infectious phases result in the production of $\mathrm{T}$ antigen, a viral byproduct, which may play a role in oncogenic transformation. This common trait has been used to develop diagnostic tests that identify $\mathrm{T}$ antigen in tissue and serum as a marker of adenoviral infection [1-3]. 


\section{Clinical Presentation}

As mentioned above, some serotypes and subgroups have been linked to different clinical presentations and vary by age. Most primary infections with adenovirus usually occur during infancy and early childhood $[2,4]$. Serotypes $1,2,5$, and 6 have been isolated from the lymphoepitheial and nasopharyngeal tissue of children, and have been linked to very low-grade infection (likely from latent replication) [4]. Serotypes 3, 4, and 7 have been linked to acute upper and lower respiratory tract infections in adults [2, 4].

The usual clinical presentation includes upper respiratory symptoms, rhinorrhea, pharyngitis, fevers, conjunctivitis, and cough, with lower respiratory tract symptoms [2]. The severity and extent of the clinical symptoms depend on the serotype and clinical syndrome $[2,4]$. The usual incubation period ranges from 5 to 6 days, but can take as long as 10 days $[2,4]$. The usual complications associated with lower respiratory tract symptoms are bacterial pneumonia, effusion, and superinfected bacterial empyema [2].

There are also reports of epidemic outbreaks of certain serotypes in various populations, including immunocompromised individuals [5], military recruits who have had serious lower respiratory tract infections (associated with serotypes $4,7,14$, and 21) [1, 6-8], and residential nursing homes (serotype 35) [9]. More recently, reports have been published of severe adenoviral respiratory infections associated with acute respiratory distress syndrome (ARDS) in immunocompetent adults [10•]. This clinical presentation has also been reported in two fatal cases of pneumonia in neonates, associated with serotypes 11 and 35 [11, 12]. Table 1 lists the most common respiratory clinical syndromes and their associated serotypes.

The ability of a host to clear adenovirus infection and develop protective serologic immunity is largely dependent on responses mediated by $\mathrm{T}$ cells $[2,4]$. Also, the presence of serologic immunity to one serogroup or serotype does not provide cross-protection $[2,4]$. Hence, healthy individuals have the ability to suffer from several adenoviral

Table 1 Adenoviral respiratory clinical syndromes and associated serotypes

\begin{tabular}{|c|c|}
\hline Clinical syndrome & $\begin{array}{l}\text { Frequently isolated } \\
\text { adenovirus serotype }\end{array}$ \\
\hline Upper respiratory tract infection & $\mathbf{1}, \mathbf{2}, \mathbf{3}, 5,7$ \\
\hline Lower respiratory tract infection & $3,4,7,14,21,35$ \\
\hline Pharyngoconjunctival fever & $2, \mathbf{3}, 4,7,14$ \\
\hline Epidemic keratoconjunctivitis & $3, \mathbf{8}, \mathbf{1 9}, 37$ \\
\hline
\end{tabular}

${ }^{a}$ All serotypes listed are those that have been identified in each clinical syndrome; serotypes in bold are the most commonly isolated serotypes infections in a lifetime. Individuals with impaired T-cell function (eg, stem-cell transplantation, graft-versus-host disease, and HIV infection) have been recognized to be at higher risk for severe local and disseminated infection $[4$, 13-16].

\section{Upper and Lower Respiratory Tract Infections}

Most cases of adenoviral respiratory infections initially present as a mild upper respiratory illness or bronchitis that is usually self-limited. In pediatric patients, about $5 \%$ to $10 \%$ of all lower respiratory tract infections are reported to be associated with adenovirus $[1,3,4]$. Although most are benign, the disease course may be more progressive and severe, particularly when less common serotypes are involved. In neonates, there have been case reports of fatal adenoviral pneumonia associated with serotypes 11 and 35 $[11,12]$. This variation in clinical presentation with different serotypes was also reported in immunocompetent adults. Hakim and Tleyjeh [10॰] described a case of severe adenoviral pneumonia in an immunocompetent adult that progressed to ARDS with hemorrhagic conjunctivitis. This report reviewed the literature and identified 14 other cases of severe adenoviral pneumonia in immunocompetent adults, suggesting that this serotype may have more significant clinical implications in healthy adults and adolescents $\left[10^{\bullet}\right]$.

Epidemic outbreaks of adenovirus lower respiratory tract infection, the acute respiratory disease of recruits, classically presents with cough, rhinitis, sore throat, and fever that can last for 3 to 5 days [1]. Physical examination findings are most notable for rales and/or rhonchi and mild pharyngitis. Chest radiographs can show mild, bibasilar, ground-glass opacities that correlate with the clinical examination, or can be completely normal [1]. In most immunocompetent children and adults, the infection is usually self-limited or asymptomatic with no apparent sequela. As stated above, the most common serotypes associated with this infection in young adults are 3,4 , and 7 [1].

The initial studies by Dingle and Langmuir [1, 17] detailed the classic outbreaks of adenoviral infection that occurred almost exclusively in military recruits in the 1940s and 1950s (known as acute respiratory disease of recruits). They detailed the epidemiology, natural history, and infectivity of the previously unknown causative agentsadenovirus serotypes 4 and 7-which had a tremendous impact on troop strength during basic training in Fort Bragg, NC [1, 17]. These serotypes were found to cause respiratory tract illnesses almost exclusively in military recruits, and only minimal impact in the general population or among pediatric patients [1, 17]. Outbreaks of adenovirus-associated acute respiratory disease had an 
attack rate of about $80 \%$ of new recruits, and a hospitalization rate of $20 \%$ - a substantial negative impact on military forces during basic training [1, 17].

The epidemiologic work by Dingle and Langmuir [17] was the impetus for the development of the live-oral attenuated adenovirus vaccine by military infectious disease experts and the NIH in the 1960s $[1,18]$. The vaccine was very effective, resulting in a $50 \%$ to $60 \%$ reduction of respiratory illness in new recruits $[1,18]$. This vaccine was in routine use at military training sites by 1971 , and targeted serotypes 4 and 7, the two most common serotypes associated with severe disease. However, in the late 1990s, the vaccine was no longer manufactured and administered to military recruits. This circumstance resulted in a resurgence of adenovirus respiratory tract disease with the familiar serotypes 4 and 7, as well as the emergence of two new serotypes, 14 and 21, among military recruits across the United States $[6,19 \bullet, 20 \bullet]$.

In 2007, a well-documented outbreak of serotype 14 occurred among military recruits and affected $48 \%$ of basic trainees, resulting in many hospitalizations and intensive care admissions, and one death [20•]. During this time, an increase was noted in adenovirus serotypes 3, 7, 21, and most commonly serotype 14 in military training facilities across the United States [19॰]. Studies performed in the civilian population in the local area showed that among pediatric isolates, the most common serotypes isolated were adenoviruses 1,2 , and 3 , rather than the serotypes seen in the basic training facilities [21]. However, in Oregon, serotype 14 was found in $60 \%$ of adenovirus isolates; many of these patients were older with underlying chronic diseases and immunosuppression, which ultimately resulted in hospitalization [22••]. The overall impression from these outbreaks suggests that certain clinical presentations and populations with adenoviral respiratory infections should alert physicians to the potential of more severe illness and the need for closer monitoring and preventive measures.

More recently, as advanced medical care has led to higher numbers of patients with periods of prolonged, suppressed, cell-mediated immunity, severe adenoviral lower respiratory infections have become a significant cause of morbidity and mortality among these patients. This group includes stem-cell transplant recipients, latestage HIV-infected individuals, and those receiving solid organ transplants $[5,13-16]$. These infections are from either the reactivation of latent virus or the recent acquisition of a new serotype. Clinically, they can range from asymptomatic viral shedding to severe pneumonia or ARDS. Other clinical manifestations described in these patients include fulminant hepatitis, gastroenteritis, hemorrhagic cystitis, and encephalitis [5, 13-16]. Studies have reported that the overall incidence of acute adenovirus infection in stem-cell transplant patients ranged from $6 \%$ to
$30 \%[5,14,23]$. In addition, clinical presentations ranged from hemorrhagic cystitis to severe pneumonia or upper respiratory infections $[5,22 \bullet \cdot]$. Overall, the risk factors associated with severe infection were younger age, presence of graft-versus-host disease, receipt of an allogeneic bone marrow transplant, and receipt of preparatory total body irradiation prior to transplant $[5,23,24]$. The morbidity and mortality associated with this infection in immunocompromised individuals has led to the use of cidofovir treatment regimens and other supportive measures for patients with acute adenoviral infection [2, 5, 23, 24].

\section{Pharyngoconjunctival Fever}

Pharyngoconjunctival fever was one of the first described clinical entities to be associated with concurrent evidence of acute adenovirus infection $[1,2,25]$. It was initially described in 1956, as part of an epidemic outbreak with serotype 3 [25]. It typically presents in children, and has been associated with outbreaks in summer camps, orphanages, and residential neighborhoods. These outbreaks were caused by a contaminated swimming pool and crowded living conditions $[25,26]$.

The classic presentation is the acute onset of high fever, pharyngitis, and preauricular or cervical lymphadenitis, followed by nonpurulent follicular conjunctivitis. The illness is usually self-limited, lasting 5 to 7 days $[2,25$, 26]. Fortunately, it is rarely associated with the more severe complications of lower respiratory tract disease or bacterial superinfection $[2,25,26]$. The clinical syndrome can mimic other infectious processes such as influenza, epidemic keratoconjunctivitis, herpes, herpangina, and other viral infections. This characteristic has led to difficulty in making the diagnosis during early presentation. The conjunctivitis associated with this syndrome is usually self-limited, resolving within a few days. It rarely leads to keratitis or other ophthalmologic sequela $[2,26]$.

\section{Epidemic Keratoconjunctivitis}

Epidemic keratoconjunctivitis (EKC) is a more serious clinical condition associated with adenoviral infection. It has been reported as a major pathogen associated with small community epidemics and nosocomial outbreaks involving hospitalized adults, residential nursing homes, and neonatal intensive care units [27-29]. The clinical presentation in adults is described as acute onset of pain, photophobia, increased lacrimation, and conjunctival injection. Some patients have also described the sensation of a foreign body within the involved eye [2, 27-29]. Infants and neonates tend to present with conjunctival injection, 
increased lacrimation, and periorbital edema. The lack of ability to detect pain or photophobia in this patient population has been associated with delays in diagnosis, contributing to nosocomial outbreaks in neonatal intensive care units [27-29]. The most common serotypes associated with EKC are 8, 19, and 37 [27-29]. More recently, adenovirus $22 / \mathrm{H} 8$, a new hybrid adenovirus, has also been associated with EKC among patients who were examined with contaminated ophthalmologic equipment [30].

Most of these outbreaks have been associated with contaminated ophthalmologic equipment, droplet and respiratory spread among family members, and secondary spread to healthcare workers $[27,28,30]$. As a result, there has been greater focus on early identification of these infections via polymerase chain reaction (PCR) and/or direct florescent antigen testing, and early and effective isolation and infection-control methods for all identified patients $[27,28]$. The incubation period for $\mathrm{EKC}$ is 4 to 24 days, and the usual course is a self-limited illness [27-29]. In some cases, it may progress to a deeply established keratitis, which can persist for months and can lead to visual disturbances or significant corneal damage [31].

\section{Diagnosis}

The diagnosis of adenovirus infection is usually presumptive, based on clinical syndromes and symptoms. Testing for the presence of the virus in respiratory secretions, serum, urine, stool, and conjunctival scrapings can be performed via culture, real-time PCR, or antigen detection $[2,32,33 \bullet]$. However, all of these tests have limitations. The virus has the ability to persist as a latent subclinical or chronic infection, which does not necessarily prove its role as the causative agent when it is detected [32, 33•]. In addition, acute and convalescent serum antibody titers can be measured, and show a fourfold rise following acute illness. Culture of adenovirus usually takes 5 to 7 days, although recovery is a challenge because only $50 \%$ to $70 \%$ of samples yield positive results [2]. However, real-time PCR has a much higher sensitivity and provides results within 1 to 2 days [2, 32, 33•]. The rapidity of real-time PCR is extremely helpful in the clinical setting, especially in outbreaks and epidemics. Recently, Metzgar et al. [34] described the increased utility of real-time PCR, especially in detection of pathogenic serotype 14. Their study showed that PCR had a higher sensitivity and specificity for serotype 14, with no known cross-reactivity with other respiratory viral pathogens [34].

Antigen testing is performed via immunofluorescent or enzyme-linked immunosorbant assays. These tests are less expensive than PCR, and provide results within 2 to 3 days [2]. However the sensitivity and specificity of these tests is low, requiring most negative tests to be confirmed by traditional viral culture, which can take another 4 to 5 days and makes them less useful in clinical settings.

\section{Treatment}

The treatment of adenovirus respiratory tract infections consists primarily of supportive care. To date, there is no approved antiviral therapy in immunocompetent patients, especially because the illness is usually self-limited. Anecdotal reports exist of ganciclovir and ribavirin being used to treat severe adenoviral disease in immunocompromised individuals, but these drugs have shown variable success [35].

Currently, cidofovir is the only recognized, well-studied antiviral agent that has shown some success in adenoviral respiratory tract infections. It is commonly used, with moderate success, in immunocompromised patients with acute adenoviral infection [36]. In a previous outbreak of serotype 14 in a civilian population, cidofovir was tried in some patients and resulted in variable success. Not all patients who were hospitalized received cidofovir; however, of the six who did, four survived their infection [22••]. The major limitation of cidofovir therapy is the high risk of nephrotoxicity, which limits its use and duration of therapy, especially in high-risk or immunocompromised patients [36]. Some protocols use large fluid boluses to help prevent these consequences; however, there are limitations to their use, and they are not entirely successful in preventing this complication. More recently, a study assessing the efficacy of cidofovir with and without intravenous immunoglobulin showed some benefit in the group that received combination therapy with immunoglobulin and cidofovir in stemcell and solid organ transplant patients [37]. Overall, the nephrotoxicity and lack of definitive clinical trials to show efficacy with cidofovir therapy in adenovirus infection has deterred its routine use in patients.

\section{Prevention}

Prevention of outbreaks and epidemics of adenoviral infections are primarily mediated by good infectioncontrol and hygiene policies. Adenoviruses are spread by respiratory droplets or by the fecal-oral route [2, 38]. Therefore, patients admitted with suspected or confirmed adenovirus infection should be maintained on droplet and contact precautions.

As mentioned earlier, the average incubation period is 5 to 6 days but can be as long as 10 days [2, 38]. This long incubation has contributed to community outbreaks in close-contact living conditions, such as in nursing homes 
and among military recruits [28]. As noted above, the nonenveloped structure of adenovirus enables it to evade destruction by detergents and alcohol-based solutions, with a resultant impact on infection-control measures and the modalities used to eradicate virus colonization in hospital rooms and other medical settings [2, 27, 28].

The discovery of adenovirus serotypes 4 and 7 as the causative agent of acute respiratory disease in military recruits in 1953 led to an understanding of its clinical and financial impact on the US military [1]. These findings prompted further investigations by NIH and military infectious disease experts, resulting in the discovery that adenovirus serotype 4 and 7 gastrointestinal infection produced lasting serologic immunity and protection without resulting in clinical respiratory infection. This discovery led to the development of the bivalent oral live-attenuated adenovirus vaccine $[1,39,40]$. As mentioned earlier, the vaccine had a tremendous impact on reducing infection and morbidity and mortality associated with adenovirus acute respiratory disease in military recruits [18]. However, since discontinuing production of the vaccine, serotypes $4,7,14$, and 21 have increased significantly [39, 41]. This occurrence led to the recent development of a new adenovirus oral live-attenuated vaccine by the military. The first published reports from the phase 1 trial of the new oral adenovirus serotype 4 and 7 vaccines in military recruits showed that the vaccine produced a good immune response with minimal side effects as compared with placebo [42•]. Viral shedding continued for 1 to 3 weeks after vaccine administration; however, the development of immunity to both serotypes was noted by 28 days [42•]. Phase 2 and 3 trials were recently completed with this vaccine, and are awaiting approval by the Food and Drug Administration for its routine use in military recruits.

\section{Conclusions}

The history of adenovirus infection and its clinical impact on various patient populations, especially military recruits, is one of the major factors that led to its identification and determination of its clinical impact in pediatric and adult patients. Since its identification in 1953, certain serotypes have been linked to particular respiratory syndromes $[1,3$, 4]. In addition, certain serotypes have received much attention for their role in community and military outbreaks and epidemics $[1,7]$. The impact of these outbreaks led to the development of the first oral live-attenuated adenovirus vaccine. This vaccine resulted in a significant decrease in epidemics and outbreaks, especially among military recruits $[1,18,40]$. However, since production of the vaccine ceased in the late 1990s, we have seen an increase of more serotypes in this population, resulting in high levels of morbidity and mortality [39]. Adenovirus has also been implicated as a major pathogen in immunocompromised individuals and is notorious for causing outbreaks in hospital and clinical settings $[14,15,22 \bullet \cdot]$. The impact of this virus on the population, in particular among military recruits, has led to recent efforts to produce a new live-oral adenovirus vaccine, which is currently completing clinical trials $[42 \cdot]$.

Acknowledgement Michael Rajnik, LtCol, USAF, MC for his assistance in editing this article.

Disclosure The opinions or assertions contained in this article are the private views of the authors and are not to be construed as reflecting the views of the Uniformed Services University of the Health Sciences, the US Army, Air Force, or the Department of Defense. No potential conflict of interest relevant to this article was reported.

\section{References}

Papers of particular interest, published recently, have been highlighted as:

- Of importance,

•• Of major importance

1. Woodward TE: The Armed Forces Epidemiological Board: Its First Fifty Years. Falls Church, VA: Center of Excellence in Military Medical Research and Education, Office of the Surgeon General, Department of the Army; 1994. Available at http:// history.amedd.army.mil/booksdocs/itsfirst50yrs/fiftyyears.html.

2. Mandell GL, Bennett JE, Dolin R: Mandell, Douglas, and Bennett's Principles and Practice of Infectious Diseases, edn 7. Philadelphia: Elsevier; 2009.

3. Rowe WP, Huebner RJ, Gillmore LK, et al.: Isolation of a cytopathogenic agent from human adenoids undergoing spontaneous degeneration in tissue culture. Proc Soc Exp Biol Med 1953, 84:570-573.

4. Brandt CD, Kim HW, Vargosko AJ, et al.: Infections in 18,000 infants and children in a controlled study of respiratory tract disease. I. Adenovirus pathogenicity in relation to serologic type and illness syndrome. Am J Epidemol 1969, 90:484-500.

5. Hale, GA, Heslop HE, Krance RA, et al.: Adenovirus infection after pediatric bone marrow transplantation. Bone Marrow Transplant 1999, 23:277-282.

6. Kolavic-Gray SA, Binn LN, Sanchez JL, et al.: Large epidemic of adenovirus type 14 infection among military trainees: epidemiological, clinical and laboratory studies. Clin Infect Dis 2002, 35:808-818.

7. Van Der Veen J, Dijkman JH: Associations of type 21 Adenovirus with acute respiratory illness in military recruits. Am J Hyg 1962, 76:149-159.

8. Dudding BA, Wagner SC, Zeller JA, et al.: Fatal pneumonia associated with adenovirus type 7 in three military trainees. $\mathrm{N}$ Engl J Med 1972, 286:1289-1292.

9. Klinger JR, Sanchez MP, Curtin LA, et al.: Multiple cases of lifethreatening adenovirus pneumonia in a mental health care center. Am J Respir Crit Care Med 1998, 157:645-549.

10. - Hakim FA, Tleyjeh IM: Severe adenovirus pneumonia in immunocompetent adults: a case report and review of the literature. 
Eur J Clin Microbiol Infect Dis 2008, 27:153-158. This article describes severe adenovirus pneumonia with ARDS in immunocompetent adults, which is an unusual clinical presentation.

11. Osamura T, Mizuta R, Yoshika H, et al.: Isolation of adenovirus type 11 from the brain of a neonate with pneumonia and encephalitis. Eur J Pediatr 1993, 152:496-499.

12. Pinto A, Beck R, Jadavji T: Fatal neonatal pneumonia caused by adenovirus type 35. Report of one case and review of the literature. Arch Pathol Lab Med 1992, 116:95-99.

13. Wigger HJ, Blanc WA: Fatal hepatic and bronchial necrosis in adenovirus infection with thymic dysplasia. N Engl J Med 1964, 275:870-874.

14. Leen AM, Rooney CM: Adenovirus as an emerging pathogen in immunocompromised patients. Br J Haematol 2005, 128:135-144.

15. Kojaoghlanian T, Flomenberg P, Horwitz MS: The impact of adenovirus infection on the immunocompromised host. Rev Med Virol 2003, 13:155-171.

16. Krilov LR, Rubin LG, Frogel M, et al.: Disseminated adenovirus infection with hepatic necrosis in patients with HIV and other immunodeficiency states. Rev Infect Dis 1990, 12:303-307.

17. Dingle JH, Langmuir AD: Epidemiology of acute respiratory disease in military recruits. Am Rev Respir Dis 1968, 97 (Suppl):1-65.

18. Top FH, Dudding BA, Russell PK, et al.: Control of respiratory disease in recruits with types 4 and 7 adenovirus vaccines. Am J Epidemiol 1971, 94:142-146.

19. - Metzgar D, Osuna M, Kajon AE, et al.: Abrupt emergence of diverse species $B$ adenoviruses at US military recruit training centers. J Infect Dis 2007, 196:1465-1473. This important article details the alarming rise in respiratory tract infections with adenovirus serotypes 4, 7, 14, and 21 in military recruits across the United States.

20. - Tate JE, Bunning ML, Lott L, et al.: Outbreak of severe respiratory disease associated with emergent human adenovirus serotype 14 at a US air force training facility in 2007. J Infect Dis May 2009, 199:1419-1426. This article focuses on the severity of adenovirus 14 in healthy military recruits, and its importance as an emerging pathogen in this population.

21. Laham FR, Jewell AM, Schoonover AL, et al.: The search for adenovirus 14 in children in Houston, Texas. Pediatr Infect Dis J 2008, 27:653-654.

22. • Lewis, PF, Schmidt MA, Lu X, et al.: A community-based outbreak of severe respiratory illness caused by human adenovirus serotype 14. J Infect Dis May 2009, 199:1427-1434. This article describes the clinical presentation and impact of a community outbreak of adenovirus 14.

23. Shields A, Hackman R, Fife K, et al.: Adenovirus infection in patients undergoing bone-marrow transplantation. N Engl J Med 1985, 312:529-533.

24. Flomenberg P, Babbit J, Drobyski W, et al.: Increasing incidence of adenovirus disease in bone marrow transplant recipients. J Infect Dis 1994, 169:775-781.

25. Aronson B, Aronson S, Sobel G, et al.: Pharyngoconjunctival fevers; report of an epidemic outbreak. AMA J Dis Child 1956, 92:596-612.

26. Bell JA: Clinical manifestation of pharyngoconjunctival fever. Am J Opthalmol 1957, 43:11-14.
27. Chaberny IE, Schnitzler P, Geiss HK, Wendt C: An outbreak of epidemic keratoconjunctivitis in a pediatric unit due to adenovirus type 8. Infect Control Hosp Epidemiol 2003, 24:514-519.

28. Buffington J, Chapman LE, Stobierski MG, et al.: Epidemic keratoconjunctivitis in a chronic care facility: risk factors and measures for control. J Am Geriatr Soc 1993, 41:1177-1181.

29. Usui N, Ookoshi H, Mori H, et al.: Clinical features of epidemic keratoconjunctivitis in 41 patients. Nippon Ganka Gakkai Zasshi 2001, 105:183-188.

30. Engelmann I, Madisch I, Pommer H, Heim A: An outbreak of epidemic keratoconjuctivitis caused by a new intermediate adenovirus 22/H8 identified by molecular typing. Clin Infect Dis 2006, 43:e64-e66.

31. Murrah WF: Epidemic keratoconjunctivitis. Ann Ophthalmol 1988, 20:36-38.

32. Damen M, Minnaar R, Glasius P, et al.: Real-time PCR with an internal control for detection of all known human adenovirus serotypes. J Clin Microbiol 2008, 46:3997-4003.

33. - Wong S, Pabbaraju K, Pang XL, et al.: Detection of a broad range of human adenoviruses in respiratory tract samples using a sensitive multiplex real-time PCR assay. J Med Virol 2008, 80:856-865. This article shows the utility and sensitivity of realtime PCR in detecting most clinically relevant adenovirus serotypes.

34. Metzgar D, Skochko G, Gibbins C, et al.: Evaluation and validation of a real-time PCR assay for detection and quantitation of human adenovirus 14 from clinical samples. PLoS One 2009, 4:e7081.

35. Bruno B, Gooley T, Hackman RC, et al.: Adenovirus infection in hematopoietic stem cell transplantation: effect of ganciclovir and impact on survival. Biol Blood Marrow Transplant 2003, 9:341352 .

36. Legrand F, Berrebi D, Houhou N, et al: Early diagnosis of adenovirus infection and treatment with cidofovir after bone marrow transplantation in children. Bone Marrow Transplant 2001, 27:621-626.

37. Saquib R, Melton LB, Chandrakantan A, et al.: Disseminated adenovirus infection in renal transplant recipients: the role of cidofovir and intravenous immunoglobulin. Transpl Infect Dis 2009 (Epub ahead of print).

38. Lessler J, Reich NG, Brookmeyer R, et al.: Incubation periods of acute respiratory viral infections: a systemic review. Lancet Infect Dis 2009, 9:291-300.

39. Russell KL, Hawksworth AW, Ryan MAK, et al.: Vaccinepreventable adenoviral respiratory illness in US military recruits, 1999-2004. Vaccine 2006, 24:2835-2842.

40. Hilleman MR: Efficacy of and indications for use of adenovirus vaccine. Am J Public Health Nations Health 1958, 48:153-158.

41. Kolavic-Gray SA, Binn LN, Sanchez JL, et al: Large epidemic of adenovirus type 4 infection among military trainees: epidemiological, clinical and laboratory studies. Clin Infect Dis 2002, 35:808-818.

42. - Lyons A, Longfield J, Kuschner R, et al.: A double-blinded, placebo-controlled study of the safety and immunogenicity of live, oral type 4 and type 7 adenovirus vaccines in adults. Vaccine 2008, 26:2890-2898. This article discusses the results of the new live, oral adenovirus vaccines during their phase 1 clinical trials. 